\title{
New Exponential Stability Conditions of Switched BAM Neural Networks with Delays
}

\author{
Yi Yang ${ }^{1,2}$ \\ ${ }^{1}$ Reliability and Systems Engineering School, Beihang University, Beijing 100191, China \\ ${ }^{2}$ China Astronaut Research and Training Center, Beijing 100094, China \\ Correspondence should be addressed to Yi Yang; youngyi123@126.com
}

Received 2 April 2013; Accepted 8 April 2013

Academic Editor: Guangdeng Zong

Copyright (c) 2013 Yi Yang. This is an open access article distributed under the Creative Commons Attribution License, which permits unrestricted use, distribution, and reproduction in any medium, provided the original work is properly cited.

\begin{abstract}
The exponential stability problem is considered in this paper for discrete-time switched BAM neural networks with time delay. The average dwell time method is introduced to deal with the exponential stability analysis of the systems for the first time. By constructing a new switching-dependent Lyapunov-Krasovskii functional, some new delay-dependent criteria are developed, which guarantee the exponential stability. A numerical example is provided to demonstrate the potential and effectiveness of the proposed algorithms.
\end{abstract}

\section{Introduction}

It is well known that bidirectional associative memory (BAM) neural networks have been proposed by Kosko $[1,2]$, which include two layers: the $X$-layer and the $Y$-layer. The neurons in one layer are fully interconnected to the neurons in another layer. Recently, the dynamics analysis for BAM neural networks has received much attention due to their extensive applications in pattern recognition, solving optimization, automatic control engineering, and so forth. It is known that time delay, which will inevitably occur in the communication owing to the unavoidable finite switching speed of amplifiers, is the main cause of instability and poor performance of neural networks. Hence, it is of great importance to study the stability of BAM neural networks with time delay. Many asymptotic or exponential stability conditions for BAM neural networks with time delay were developed, see, for example [3-10] and the references therein.

On the other hand, switched systems are an important class of hybrid dynamical systems which consist of a family of continuous-time or discrete-time subsystems and a rule that orchestrates the switching among them. Switched systems provide a natural and convenient unified framework for mathematical modeling of many physical phenomena and practical applications such as autonomous transmission systems, computer disc driver, room temperature control, power electronics, and chaos generators, to name a few. Lots of valuable results concerning the stability analysis and stabilization for linear or nonlinear hybrid and switched systems were established, see, for example [11-14] and the references cited therein.

Recently, the switched neural networks, whose individual subsystems are a set of neural networks, have found their applications in the field of high-speed signal processing and artificial intelligence. Many researchers have been devoted to studying the stability issues for switched neural networks; see, for example, [15-17]. In [15], by using switched Lyapunov function method and a generalized Halanay inequality technique, the authors illustrated the asymptotic and exponential stability conditions for hybrid impulsive and switching Hopfield neural networks. While the switched Hopfield neural networks with time-varying delay were considered in [16], a robust stability condition was proposed based on the Lyapunov-Krasovskii functional approach. By combining Cohen-Grossberg neural networks with an arbitrary switching rule, the model of the switched Cohen-Grossberg neural networks with mixed time-varying delays was established in [17], and the robust stability criteria were established for these systems. However, all these results are related to the continuous-time switched neural networks. To the best of 
the authors' knowledge, stability issues of the discrete-time switched neural networks have not been fully investigated to date. Particularly for the exponential stability analysis of the discrete-time switched BAM neural networks under some constrained switching, few results have been available in the literature so far, which motivates us to carry out the present study.

In this paper, the exponential stability analysis of discretetime switched BAM neural networks with time delay is considered. To begin with, the mathematical model of the discrete-time switched BAM neural networks with time delay is established. Then by constructing a new switchingdependent Lyapunov-Krasovskii functional, some sufficient criteria are developed to guarantee the discrete-time switched BAM neural networks to be exponentially stable based on the average dwell time approach and finite sum inequality technology. Finally, A numerical example is provided to demonstrate the potential and effectiveness of the proposed algorithms.

Notations. In this paper, we use $A>0(A<0)$ to denote a positive- (negative-) definite matrix $A ; A^{T}$ represents the transpose of matrix $A ; \lambda_{M}(\cdot)$ (resp., $\lambda_{m}(\cdot)$ ) means the maximum (resp., minimum) eigenvalue of $(\cdot)$. Let $\mathbb{R}$ denote the set of real numbers; $\mathbb{R}^{n}$ denotes the $n$-dimensional Euclidean space; $\mathbb{R}^{n \times m}$ is the set of all $n \times m$ real matrices; $\mathbb{R}^{+}$denotes the set of $\{0,1,2, \ldots\} . \mathcal{N}=\{1,2, \ldots, N\}$ means a set of positive integers; $\mathbb{N}=\{1,2, \ldots, n\}$. The notation $\operatorname{diag}(\cdot)$ denotes a diagonal matrix. For given $\tau>0$ and $\theta \in[-\tau, 0],\|x(t)\|$ denotes vector norm defined by $\|x(t)\|=\sup _{-\tau \leq \theta \leq 0} \| x(t+$ $\theta) \|$. Matrices, if their dimensions are not explicitly stated, are assumed to have compatible dimensions for algebraic operations.

\section{Problem Formulation and Preliminaries}

In this section, firstly, we will establish the model of discretetime switched BAM neural networks. Consider the following discrete-time BAM neural networks with time delay $\left(\Sigma_{1}\right)$ :

$$
\begin{aligned}
& \tilde{x}_{p}(k+1) \\
& \quad=a_{p} \tilde{x}_{p}(k)+\sum_{q=1}^{n} w_{q p} \tilde{f}_{q}\left(\tilde{y}_{q}(k-d)\right)+I_{p}, \quad p \in \mathbb{N}, \\
& \tilde{y}_{q}(k+1) \\
& \quad=b_{q} \tilde{y}_{q}(k)+\sum_{p=1}^{n} v_{p q} \tilde{g}_{p}\left(\tilde{x}_{p}(k-\tau)\right)+J_{q}, \quad q \in \mathbb{N},
\end{aligned}
$$

where $\tilde{x}_{p}(k), \tilde{y}_{q}(k)$ are states of the $p$ th neuron from the neural field $F_{X}$ and the $q$ th neuron from the neural field $F_{Y}$ at time $k$, respectively. $a_{p}, b_{q} \in(0,1)$ describe the stability of internal neuron processes on the $X$-layer and the $Y$-layer, respectively. $w_{q p}, v_{p q}$ are constants and denote the synaptic connection weights. $\widetilde{f}_{q}(\cdot)$ and $\tilde{g}_{p}(\cdot)$ denote the activation functions of the $q$ th neuron from the neural field $F_{Y}$ and the $p$ th neuron from the neural field $F_{X}$, respectively. $I_{p}$ and $J_{q}$ are the external constant inputs from outside of the network acting on the $p$ th neuron from the neural field $F_{X}$ and the $q$ th neuron from the neural field $F_{Y}$, respectively. $d$ and $\tau$ are constant delays.

The system $\left(\Sigma_{1}\right)$ can be rewritten as the vector form $\left(\Sigma_{2}\right)$ :

$$
\begin{aligned}
& \tilde{x}(k+1)=A \tilde{x}(k)+W^{T} \tilde{f}(\tilde{y}(k-d))+I, \\
& \tilde{y}(k+1)=B \tilde{y}(k)+V^{T} \tilde{g}(\tilde{x}(k-\tau))+J,
\end{aligned}
$$

where

$$
\begin{gathered}
\tilde{x}(k)=\left[\tilde{x}_{1}(k), \tilde{x}_{2}(k), \ldots, \tilde{x}_{n}(k)\right]^{T}, \\
\tilde{y}(k)=\left[\tilde{y}_{1}(k), \tilde{y}_{2}(k), \ldots, \tilde{y}_{n}(k)\right]^{T}, \\
A=\operatorname{diag}\left(a_{1}, a_{2}, \ldots, a_{n}\right), \\
B=\operatorname{diag}\left(b_{1}, b_{2}, \ldots, b_{n}\right), \\
W=\left(w_{q p}\right)_{n \times n}, \quad V=\left(v_{p q}\right)_{n \times n},
\end{gathered}
$$

$\tilde{f}(\tilde{y}(k))$

$$
=\left[\tilde{f}_{1}\left(\tilde{y}_{1}(k)\right), \tilde{f}_{2}\left(\tilde{y}_{2}(k)\right), \ldots, \tilde{f}_{n}\left(\tilde{y}_{n}(k)\right)\right]^{T}
$$

$\tilde{g}(\tilde{x}(k))$

$$
\begin{aligned}
& =\left[\widetilde{g}_{1}\left(\tilde{x}_{1}(k)\right), \widetilde{g}_{2}\left(\tilde{x}_{2}(k)\right), \ldots, \widetilde{g}_{n}\left(\tilde{x}_{n}(k)\right)\right]^{T}, \\
I & =\left[I_{1}, I_{2}, \ldots, I_{n}\right], \quad J=\left[J_{1}, J_{2}, \ldots, J_{n}\right] .
\end{aligned}
$$

Throughout this paper, we always assume the following.

$\left(\mathrm{G}_{1}\right)$ The neurons activation functions $\tilde{f}_{q}(\cdot)$ and $\widetilde{g}_{p}(\cdot)(p, q \in \mathbb{N})$ are bounded on $\mathbb{R}$.

$\left(\mathrm{G}_{2}\right)$ There exist constants $\ell_{q}^{(1)}>0$ and $\ell_{p}^{(2)}>0$ such that

$$
\begin{array}{r}
\left|\tilde{f}_{q}\left(\xi_{1}\right)-\tilde{f}_{q}\left(\xi_{2}\right)\right| \leq \ell_{q}^{(1)}\left|\xi_{1}-\xi_{2}\right|, \\
\left|\tilde{g}_{p}\left(\xi_{1}\right)-\tilde{g}_{p}\left(\xi_{2}\right)\right| \leq \ell_{p}^{(2)}\left|\xi_{1}-\xi_{2}\right|, \\
\forall \xi_{1}, \xi_{2} \in \mathbb{R}, p, q \in \mathbb{N} .
\end{array}
$$

Then, under the assumptions $\left(G_{1}\right)$ and $\left(G_{2}\right)$, system $\left(\Sigma_{2}\right)$ has at least one equilibrium.

Now, we shift equilibrium point $\tilde{x}^{*}=\left[\begin{array}{lll}\tilde{x}_{1}^{*} & \tilde{x}_{2}^{*} & \ldots\end{array}\right.$ $\left.\tilde{x}_{n}^{*}\right], \tilde{y}^{*}=\left[\begin{array}{llll}\tilde{y}_{1}^{*} & \tilde{y}_{2}^{*} & \cdots & \tilde{y}_{n}^{*}\end{array}\right]$ of system $\left(\Sigma_{2}\right)$ to the origin. Let $x(k)=\tilde{x}(k)-\tilde{x}^{*}, y(k)=\widetilde{y}(k)-\tilde{y}^{*}$; then the system $\left(\Sigma_{2}\right)$ can be transformed to the following system $\left(\Sigma_{3}\right)$ :

$$
\begin{aligned}
& x(k+1)=A x(k)+W^{T} f(y(k-d)), \\
& y(k+1)=B y(k)+V^{T} g(x(k-\tau)),
\end{aligned}
$$


where

$$
\begin{gathered}
x(k)=\left[x_{1}(k), x_{2}(k), \ldots, x_{n}(k)\right]^{T}, \\
y(k)=\left[y_{1}(k), y_{2}(k), \ldots, y_{n}(k)\right]^{T}, \\
f(y(k))=\left[f_{1}\left(y_{1}(k)\right), f_{2}\left(y_{2}(k)\right), \ldots, f_{n}\left(y_{n}(k)\right)\right]^{T}, \\
g(x(k))=\left[g_{1}\left(x_{1}(k)\right), g_{2}\left(x_{2}(k)\right), \ldots, g_{n}\left(x_{n}(k)\right)\right]^{T}, \\
f_{q}\left(y_{q}(k)\right)=\tilde{f}_{q}\left(\tilde{y}_{q}(k)\right)-\tilde{f}_{q}\left(\tilde{y}_{q}^{*}\right), \quad q \in \mathbb{N}, \\
g_{p}\left(x_{p}(k)\right)=\tilde{g}_{p}\left(\tilde{x}_{p}(k)\right)-\tilde{g}_{p}\left(\tilde{g}_{p}^{*}\right), \quad p \in \mathbb{N} .
\end{gathered}
$$

Obviously, the activation functions $f_{q}(\cdot)$ and $g_{p}(\cdot)$ satisfy the following conditions.

$\left(\mathrm{G}_{3}\right)$ There exist constants $\ell_{q}^{(1)}>0$ and $\ell_{p}^{(2)}>0$ such that

$$
\begin{aligned}
\left|f_{q}(\xi)\right| \leq \ell_{q}^{(1)}|\xi|, & \left|g_{p}(\xi)\right| \leq \ell_{p}^{(2)}|\xi|, \\
& \forall \xi \in \mathbb{R}, p, q \in \mathbb{N} .
\end{aligned}
$$

With the rapid development of intelligent control, hybrid systems have been investigated due to their extensive applications. In recent years, considerable efforts have been focused on analysis and design of switched systems. The discretetime switched system can be characterized by the following difference equation $\left(\Sigma_{4}\right)$ :

$$
x(k+1)=\Gamma_{\sigma(k)} x(k),
$$

where $\sigma(k)$ is a switching signal which takes its values in the finite set $\mathcal{N}=\{1,2, \ldots, N\} . \Gamma_{\sigma(k)}=\Gamma_{i}$, when $\sigma(k)=i$, are the functions of the switching signals.

Combining the theories of switched systems and discretetime BAM neural networks, the discrete-time switched BAM neural networks can be formulated as the following system $(\Sigma)$ :

$$
\begin{gathered}
x(k+1)=A_{\sigma(k)} x(k)+W_{\sigma(k)}^{T} f(y(k-d)), \\
y(k+1)=B_{\sigma(k)} y(k)+V_{\sigma(k)}^{T} g(x(k-\tau)),
\end{gathered}
$$

where $\sigma(k)$ is a switching signal which takes its values in the finite set $\mathcal{N}=\{1,2, \ldots, N\}$.

For the discrete-time switched BAM neural networks $(\Sigma)$, we have the following assumptions.

$\left(\mathrm{H}_{1}\right)$ The initial value is $x(s)=\phi(s), y(s)=\psi(s), s \in$ $[-h, 0]$, where $h=\max \{d, \tau\}$.

$\left(\mathrm{H}_{2}\right)$ There exist matrices $L_{1}>0$ and $L_{2}>0$ such that

$$
|f(\xi)| \leq L_{1}|\xi|, \quad|g(\xi)| \leq L_{2}|\xi|, \quad \forall \xi \in \mathbb{R},
$$

where $L_{1}=\operatorname{diag}\left(\ell_{1}^{(1)}, \ell_{2}^{(1)}, \ldots, \ell_{n}^{(1)}\right)$ and $L_{2}=$ $\operatorname{diag}\left(\ell_{1}^{(2)}, \ell_{2}^{(2)}, \ldots, \ell_{n}^{(2)}\right)$.

$\left(\mathrm{H}_{3}\right)$ Switching sequence is defined as $\zeta=\left\{\left[\begin{array}{ll}x_{k_{0}} & y_{k_{0}}\end{array}\right]^{T}\right.$; $\left.\left(i_{0}, k_{0}\right),\left(i_{1}, k_{1}\right), \ldots,\left(i_{m}, k_{m}\right), \ldots, \mid i_{m} \in \mathcal{N}, m \in \mathbb{R}^{+}\right\}$. When $k \in\left[k_{m}, k_{m+1}\right)$, the $k_{m}$ th subsystem is activated and the states of system $(\Sigma)$ do not jump when switch occurs.
Remark 1. By combining the switched systems theory and the discrete-time BAM neural networks model, the mathematical model of discrete-time switched BAM neural networks is introduced as above. A set of discrete-time BAM neural networks with time delay are used as the subsystems, and an arbitrary switching rule is assumed to coordinate the switching between these neural networks.

To present the main results of this paper more precisely, the following definitions and lemmas are introduced, which will be essential for the later development.

Definition 2 (see [12]). For any $k \geq k_{0}$ and any switched signal $\sigma(\varsigma), k_{0} \leq \varsigma<k$, let $N_{\sigma}$ denote the switching numbers of $\sigma(\varsigma)$ during the interval $\left[k_{0}, k\right]$. If there exist $N_{0} \geq 0$ and $T_{a}>0$ such that $N_{\sigma}\left(k_{0}, k\right) \leq N_{0}+\left(k-k_{0}\right) / T_{a}$, then $T_{a}$ and $N_{0}$ are called average dwell time and the chatter bound, respectively.

Definition 3. The discrete-time switched BAM neural network $(\Sigma)$ is said to be exponentially stable if its solution satisfies

$$
\|x(k)\|^{2}+\|y(k)\|^{2} \leq K\left(\|\phi\|_{L}^{2}+\|\psi\|_{L}^{2}\right) \lambda^{-\left(k-k_{0}\right)}, \quad \forall k \geq k_{0},
$$

for any initial condition $\left(k_{0}, \phi\right) \in \mathbb{R}^{+} \times C^{n}$ and $\left(k_{0}, \psi\right) \in$ $\mathbb{R}^{+} \times C^{n} \cdot\|\phi\|_{L}=\sup _{k_{0}-h \leq \ell \leq k_{0}}\|\phi(\ell)\|$, and $\|\psi\|_{L}=$ $\sup _{k_{0}-h \leq \ell \leq k_{0}}\|\psi(\ell)\|, h=\max \{d, \tau\} . K>0$ is the decay coefficient, and $\lambda>1$ is the decay rate.

Remark 4. Without loss of generality, in this paper, we assume $N_{0}=0$ for simplicity as commonly used in the literature.

Remark 5. Based on the definition of exponential stability for BAM neural networks in [5] and the definition of exponential stability for switched systems in [13], we give the above definition of exponential stability for discrete-time switched BAM neural networks.

Lemma 6 (the Schur complement [18]). For any symmetric matrix $S=\left[\begin{array}{ll}S_{11} & S_{12} \\ S_{12}^{T} & S_{22}\end{array}\right]<0$, the following conditions are equivalent:
(i) $S_{11}<0$, and $S_{22}-S_{12}^{T} S_{11}^{-1} S_{12}<0$,
(ii) $S_{22}<0$, and $S_{11}-S_{12} S_{22}^{-1} S_{12}^{T}<0$.

Lemma 7 (finite sum inequality [13]). For any constant mat$\operatorname{rix} Y=\left[\begin{array}{ll}M_{1} & M_{2}\end{array}\right] \in \mathbb{R}^{n \times 2 n}, R>0, h \geq 0$, the following inequality holds:

$$
\begin{aligned}
-\sum_{j=k-h}^{k-1} \ell^{T}(j) R \ell(j) \leq & \xi^{T}(k)\left[\begin{array}{cc}
M_{1}^{T}+M_{1} & -M_{1}^{T}+M_{2} \\
* & -M_{2}^{T}-M_{2}
\end{array}\right] \\
& \times \xi(k)+h \xi^{T}(k) Y^{T} R^{-1} Y \xi(k),
\end{aligned}
$$

where $\ell(k)=x(k+1)-x(k)$ and $\xi(k)=\left[\begin{array}{ll}x^{T}(k) & x^{T}(k-h)\end{array}\right]^{T}$. 


\section{Main Result}

In this section, the exponential stability condition for the discrete-time switched BAM neural networks $(\Sigma)$ will be presented using the average dwell time method.

When $\sigma(k)=i$, we have the following subsystem $\left(\Sigma^{i}\right)$ :

$$
\begin{gathered}
x(k+1)=A_{i} x(k)+W_{i}^{T} f(y(k-d)), \\
y(k+1)=B_{i} y(k)+V_{i}^{T} g(x(k-\tau)) .
\end{gathered}
$$

Choose the Lyapunov-Krasovskii functional candidate for the subsystem $\left(\Sigma^{i}\right)$ as

$$
V_{i}(k)=V_{1 i}(k)+V_{2 i}(k)+V_{3 i}(k),
$$

where

$$
\begin{aligned}
V_{1 i}(k)= & x^{T}(k) P_{1 i} x(k)+y^{T}(k) P_{2 i} y(k), \\
V_{2 i}(k)= & \sum_{\theta=k-\tau}^{k-1} r^{\theta-k+1} x^{T}(\theta) Q_{1 i} x(\theta) \\
& +\sum_{\theta=k-d}^{k-1} r^{\theta-k+1} y^{T}(\theta) Q_{2 i} y(\theta), \\
V_{3 i}(k)= & \sum_{s=-\tau}^{-1} \sum_{\theta=k+s}^{k-1} r^{\theta-k+1} \ell_{1}^{T}(\theta) R_{1 i} \ell_{1}(\theta) \\
& +\sum_{s=-d}^{-1} \sum_{\theta=k+s}^{k-1} r^{\theta-k+1} \ell_{2}^{T}(\theta) R_{2 i} \ell_{2}(\theta), \\
& \ell_{1}(k)=x(k+1)-x(k), \\
& \ell_{2}(k)=y(k+1)-y(k) .
\end{aligned}
$$

Now we give the following theorem, which plays an important role in the derivation of the exponential stability condition for the discrete-time switched BAM neural networks $(\Sigma)$.

Theorem 8. Under the assumptions $\left(H_{1}\right)-\left(H_{3}\right)$, for given scalar $r>1$, the decay estimation

$$
V_{i}(k) \leq r^{-\left(k-k_{0}\right)} V_{i}\left(k_{0}\right)
$$

is satisfied along any trajectory of system $\left(\Sigma^{i}\right)$ if there exist matrices $P_{1 i}>0, P_{2 i}>0, Q_{1 i}>0, Q_{2 i}>0, R_{1 i}>0, R_{2 i}>$ $0, N_{1 i}, N_{2 i}, M_{1 i}, M_{2 i}, T_{1 i}>0$, and $T_{2 i}>0, i \in \mathcal{N}$, such that the following linear matrix inequality holds:

$$
\left[\begin{array}{ccc}
\Omega_{i} & \Gamma_{1 i}^{T} & \Gamma_{2 i}^{T} \\
* & -\tau r^{\tau} R_{1 i} & 0 \\
* & * & -d r^{d} R_{2 i}
\end{array}\right]<0,
$$

where

$$
\begin{aligned}
& \Omega_{i}=\left[\begin{array}{cccccc}
\Omega_{11} & \Omega_{12} & 0 & 0 & 0 & \Omega_{16} \\
* & \Omega_{22} & 0 & 0 & 0 & 0 \\
* & * & \Omega_{33} & \Omega_{34} & 0 & 0 \\
* & * & * & \Omega_{44} & \Omega_{45} & 0 \\
* & * & * & * & \Omega_{55} & 0 \\
* & * & * & * & * & \Omega_{66}
\end{array}\right], \\
& \Gamma_{1 i}=\left[\begin{array}{llllll}
\tau N_{1 i} & \tau N_{2 i} & 0 & 0 & 0 & 0
\end{array}\right], \\
& \Gamma_{2 i}=\left[\begin{array}{llllll}
0 & 0 & 0 & d M_{1 i} & d M_{2 i} & 0
\end{array}\right], \\
& \Omega_{11}=A_{i} P_{1 i} A_{i}-r^{-1} P_{1 i}+Q_{1 i} \\
& +\left(A_{i}-I\right)\left(\tau R_{1 i}\right)\left(A_{i}-I\right)+r^{-\tau}\left(N_{1 i}^{T}+N_{1 i}\right), \\
& \Omega_{12}=r^{-\tau}\left(-N_{1 i}^{T}+N_{2 i}\right), \\
& \Omega_{22}=-r^{-\tau}\left(Q_{1 i}+N_{2 i}^{T}+N_{2 i}\right)+T_{1 i}, \\
& \Omega_{33}=V_{i}\left(P_{2 i}+d R_{2 i}\right) V_{i}^{T}-L_{1 i}^{-1} T_{1 i} L_{1 i}^{-1}, \\
& \Omega_{34}=V_{i} P_{2 i} B_{i}+V_{i}\left(d R_{2 i}\right)\left(B_{i}-I\right), \\
& \Omega_{44}=B_{i} P_{2 i} B_{i}-r^{-1} P_{2 i}+Q_{2 i}+\left(B_{i}-I\right)\left(d R_{2 i}\right)\left(B_{i}-I\right) \\
& +r^{-d}\left(M_{1 i}^{T}+M_{1 i}\right) \\
& \Omega_{45}=r^{-d}\left(-M_{1 i}^{T}+M_{2 i}\right), \\
& \Omega_{55}=-r^{-d}\left(Q_{2 i}+M_{2 i}^{T}+M_{2 i}\right)+T_{2 i} \text {, } \\
& \Omega_{16}=A_{i} P_{1 i} W_{i}^{T}+\left(A_{i}-I\right)\left(\tau R_{1 i}\right) W_{i}^{T}, \\
& \Omega_{66}=W_{i}\left(P_{1 i}+\tau R_{1 i}\right) W_{i}^{T}-L_{2 i}^{-1} T_{2 i} L_{2 i}^{-1} .
\end{aligned}
$$

Proof. Calculating the differential of $V_{i}(k)$ along the trajectory of system $\left(\Sigma^{i}\right)$, we obtain

$$
\begin{aligned}
\Delta V_{1 i}(k)= & x^{T}(k+1) P_{1 i} x(k+1) \\
& +y^{T}(k+1) P_{2 i} y(k+1) \\
& -x^{T}(k) P_{1 i} x(k)-y^{T}(k) P_{2 i} y(k) \\
= & x^{T}(k+1) P_{1 i} x(k+1) \\
& -r^{-1} x^{T}(k) P_{1 i} x(k)+y^{T}(k+1) P_{2 i} y(k+1) \\
& -r^{-1} y^{T}(k) P_{2 i} y(k)+\left(r^{-1}-1\right) V_{1 i}(k),
\end{aligned}
$$




$$
\begin{array}{rlr}
\Delta V_{2 i}(k)= & -\sum_{\theta=k-d}^{k-1} r^{s-k} \ell_{2}^{T}(s) R_{2 i} \ell_{2}(s)+\left(r^{-1}-1\right) V_{3 i}(k) \\
& +\sum_{\theta=k+1-d}^{k} r^{\theta-k} y^{T}(\theta) Q_{2 i} y(\theta) & \leq \tau \ell_{1}^{T}(k) R_{1 i} \ell_{1}(k)+d \ell_{2}^{T}(k) R_{2 i} \ell_{2}(k) \\
& -\sum_{\theta=k-\tau}^{k-1} r^{\theta-k+1} x^{T}(\theta) Q_{1 i} x(\theta) & -\sum_{s=k-\tau}^{k-1} r^{-\tau} \ell_{1}^{T}(s) R_{1 i} \ell_{1}(s) \\
& -\sum_{\theta=k-d}^{k-1} r^{\theta-k+1} y^{T}(\theta) Q_{2 i} y(\theta) & -\sum_{s=k-d}^{k-1} r^{-d} \ell_{2}^{T}(s) R_{2 i} \ell_{2}(s)+\left(r^{-1}-1\right) V_{3 i}(k) .
\end{array}
$$$$
=x^{T}(k) Q_{1 i} x(k)-r^{-\tau} x^{T}(k-\tau) Q_{1 i} x(k-\tau)
$$$$
+y^{T}(k) Q_{2 i} y(k)
$$$$
-r^{-d} y^{T}(k-d) Q_{2 i} y(k-d)+\left(r^{-1}-1\right) V_{2 i}(k),
$$

Note that

$$
\begin{aligned}
& -r^{-\tau} \sum_{s=k-\tau}^{k-1} \ell_{1}^{T}(s) R_{1 i} \ell_{1}(s) \\
& \leq r^{-\tau} \eta_{1}^{T}(k)\left[\begin{array}{cc}
N_{1 i}^{T}+N_{1 i} & -N_{1 i}^{T}+N_{2 i} \\
* & -N_{2 i}^{T}-N_{2 i}
\end{array}\right] \eta_{1}(k) \\
& +r^{-\tau} \eta_{1}^{T}(k)\left[\begin{array}{c}
N_{1 i}^{T} \\
N_{2 i}^{T}
\end{array}\right] \tau R_{1 i}^{-1}\left[\begin{array}{ll}
N_{1 i} & N_{2 i}
\end{array}\right] \eta_{1}(k), \\
& -r^{-d} \sum_{s=k-d}^{k-1} \ell_{2}^{T}(s) R_{2 i} \ell_{2}(s) \\
& \leq r^{-d} \eta_{2}^{T}(k)\left[\begin{array}{cc}
M_{1 i}^{T}+M_{1 i} & -M_{1 i}^{T}+M_{2 i} \\
* & -M_{2 i}^{T}-M_{2 i}
\end{array}\right] \eta_{2}(k) \\
& +r^{-d} \eta_{2}^{T}(k)\left[\begin{array}{l}
M_{1 i}^{T} \\
M_{2 i}^{T}
\end{array}\right] d R_{2 i}^{-1}\left[\begin{array}{ll}
M_{1 i} & M_{2 i}
\end{array}\right] \eta_{2}(k),
\end{aligned}
$$$$
\Delta V_{3 i}(k)=\sum_{s=-\tau}^{-1} \sum_{\theta=k+1+s}^{k} r^{\theta-k} \ell_{1}^{T}(\theta) R_{1 i} \ell_{1}(\theta)
$$$$
+\sum_{s=-d}^{-1} \sum_{\theta=k+1+s}^{k} r^{\theta-k} \ell_{2}^{T}(\theta) R_{2 i} \ell_{2}(\theta)
$$$$
-\sum_{s=-\tau}^{-1} \sum_{\theta=k+s}^{k-1} r^{\theta-k+1} e_{1}^{T}(\theta) R_{1 i} \ell_{1}(\theta)
$$$$
-\sum_{s=-d}^{-1} \sum_{\theta=k+s}^{k-1} r^{\theta-k+1} e_{2}^{T}(\theta) R_{2 i} \ell_{2}(\theta)
$$$$
=\sum_{s=-\tau}^{-1}\left(\ell_{1}^{T}(k) R_{1 i} \ell_{1}(k)\right.
$$$$
+\sum_{\theta=k+s}^{k-1} r^{\theta-k} \ell_{1}^{T}(\theta) R_{1 i} \ell_{1}(\theta)
$$$$
\left.-r^{s} \ell_{1}^{T}(k+s) R_{1 i} \ell_{1}(k+s)\right)
$$$$
+\sum_{s=-d}^{-1}\left(\ell_{2}^{T}(k) R_{2 i} \ell_{2}(k)\right.
$$$$
+\sum_{\theta=k+s}^{k-1} r^{\theta-k} \ell_{2}^{T}(\theta) R_{2 i} \ell_{2}(\theta)
$$$$
\left.-r^{s} \ell_{2}^{T}(k+s) R_{2 i} \ell_{2}(k+s)\right)-V_{3 i}(k)
$$

where

$$
\begin{aligned}
& \eta_{1}(k)=\left[\begin{array}{c}
x(k) \\
x(k-\tau)
\end{array}\right], \quad \eta_{2}(k)=\left[\begin{array}{c}
y(k) \\
y(k-d)
\end{array}\right], \\
& x^{T}(k-\tau) T_{1 i} x(k-\tau) \\
& \quad-g^{T}(x(k-\tau)) L_{1 i}^{-1} T_{1 i} L_{1 i}^{-1} g(x(k-\tau)) \geq 0, \\
& y^{T}(k-d) T_{2 i} x(k-d) \\
& \quad-f^{T}(y(k-d)) L_{2 i}^{-1} T_{2 i} L_{2 i}^{-1} f(y(k-d)) \geq 0 .
\end{aligned}
$$

From (20) to (24), the following inequality is satisfied:

$\Delta V_{i}(k)$

$$
\begin{aligned}
\leq & {\left[\begin{array}{c}
x(k) \\
f(y(k-d))
\end{array}\right]^{T}\left[\begin{array}{c}
A_{i} \\
W_{i}
\end{array}\right] P_{1 i}\left[\begin{array}{ll}
A_{i} & W_{i}^{T}
\end{array}\right]\left[\begin{array}{c}
x(k) \\
f(y(k-d))
\end{array}\right] } \\
& -x^{T}(k) r^{-1} P_{1 i} x(k)+x^{T}(k) Q_{1 i} x(k) \\
& -x^{T}(k-\tau) r^{-\tau} Q_{1 i} x(k-\tau) \\
& +\left[\begin{array}{c}
y(k) \\
g(x(k-\tau))
\end{array}\right]^{T}\left[\begin{array}{c}
B_{i} \\
V_{i}
\end{array}\right] P_{2 i}\left[\begin{array}{ll}
B_{i} & V_{i}^{T}
\end{array}\right]\left[\begin{array}{c}
y(k) \\
g(x(k-\tau))
\end{array}\right]
\end{aligned}
$$




$$
\begin{aligned}
& -y^{T}(k) r^{-1} P_{2 i} y(k)+y^{T}(k) Q_{2 i} y(k) \\
& -y^{T}(k-d) r^{-d} Q_{2 i} y(k-d) \\
& +\left[\begin{array}{c}
x(k) \\
f(y(k-d))
\end{array}\right]^{T}\left[\begin{array}{c}
A_{i}-I \\
W_{i}
\end{array}\right] \\
& \times \tau R_{1 i}\left[\begin{array}{ll}
A_{i}-I & W_{i}^{T}
\end{array}\right]\left[\begin{array}{c}
x(k) \\
f(y(k-d))
\end{array}\right] \\
& +\left[\begin{array}{c}
y(k) \\
g(x(k-\tau))
\end{array}\right]^{T}\left[\begin{array}{c}
B_{i}-I \\
V_{i}
\end{array}\right] \\
& \times d R_{2 i}\left[\begin{array}{ll}
B_{i}-I & V_{i}^{T}
\end{array}\right]\left[\begin{array}{c}
y(k) \\
g(x(k-\tau))
\end{array}\right] \\
& +r^{-\tau} \eta_{1}^{T}(k)\left[\begin{array}{cc}
N_{1 i}^{T}+N_{1 i} & -N_{1 i}^{T}+N_{2 i} \\
* & -N_{2 i}^{T}-N_{2 i}
\end{array}\right] \eta_{1}(k) \\
& +r^{-\tau} \eta_{1}^{T}(k)\left[\begin{array}{c}
N_{1 i}^{T} \\
N_{2 i}^{T}
\end{array}\right] \tau R_{1 i}^{-1}\left[\begin{array}{ll}
N_{1 i} & N_{2 i}
\end{array}\right] \eta_{1}(k) \\
& +r^{-d} \eta_{2}^{T}(k)\left[\begin{array}{cc}
M_{1 i}^{T}+M_{1 i} & -M_{1 i}^{T}+M_{2 i} \\
* & -M_{2 i}^{T}-M_{2 i}
\end{array}\right] \eta_{2}(k)
\end{aligned}
$$

$$
\zeta(k)=\left[\begin{array}{llllll}
x^{T}(k) & x^{T}(k-\tau) & g^{T}(x(k-\tau)) & y^{T}(k) & y^{T}(k-d) & f^{T}(y(k-d))
\end{array}\right]^{T} .
$$

Therefore, from (17), we have

$$
\Delta V_{i}(k) \leq\left(r^{-1}-1\right) V_{i}(k),
$$

which implies (16) is true. This completes the proof of Theorem 8 .

In what follows, we are in a position to derive the delaydependent exponential stability condition for the discretetime switched BAM neural networks $(\Sigma)$, and the results are given in the following theorem.

Theorem 9. Under the assumptions $\left(H_{1}\right)-\left(H_{3}\right)$, for given scalars $r>1, \mu \geq 1$, the system $(\Sigma)$ is exponentially stable and ensures a decay rate $\lambda$, where $\lambda=r^{\left(-\ln \mu /\left(T_{a} \ln r\right)\right)+1}$, if there exist matrices $P_{1 i}>0, P_{2 i}>0, Q_{1 i}>0, Q_{2 i}>0, R_{1 i}>0, R_{2 i}>$ $0, N_{1 i}, N_{2 i}, M_{1 i}, M_{2 i}$, and $T_{1 i}>0, T_{2 i}>0, i \in \mathcal{N}$, such that (17) and the following inequalities hold:

$$
\begin{array}{ccc}
P_{1 \alpha} \leq \mu P_{1 \beta}, & P_{2 \alpha} \leq \mu P_{2 \beta}, & Q_{1 \alpha} \leq \mu Q_{1 \beta}, \\
Q_{2 \alpha} \leq \mu Q_{2 \beta}, & R_{1 \alpha} \leq \mu R_{1 \beta}, & R_{2 \alpha} \leq \mu R_{2 \beta}, \\
T_{a} \geq T_{a}^{*}=\text { ceil }\left[\frac{\ln \mu}{\ln r}\right], & \alpha, \beta \in \mathcal{N} .
\end{array}
$$

$$
\begin{aligned}
& +r^{-d} \eta_{2}^{T}(k)\left[\begin{array}{c}
M_{1 i}^{T} \\
M_{2 i}^{T}
\end{array}\right] d R_{2 i}^{-1}\left[\begin{array}{ll}
M_{1 i} & M_{2 i}
\end{array}\right] \eta_{2}(k) \\
& +x^{T}(k-\tau) T_{1 i} x(k-\tau) \\
& -g^{T}(x(k-\tau)) L_{1 i}^{-1} T_{1 i} L_{1 i}^{-1} g(x(k-\tau)) \\
& +y^{T}(k-d) T_{2 i} x(k-d) \\
& -f^{T}(y(k-d)) L_{2 i}^{-1} T_{2 i} L_{2 i}^{-1} f(y(k-d)) \\
& +\left(r^{-1}-1\right) V_{i}(k) \\
& =\zeta^{T}(k) \Omega_{i} \zeta(k)+r^{-\tau} \eta_{1}^{T}(k)\left[\begin{array}{l}
N_{1 i}^{T} \\
N_{2 i}^{T}
\end{array}\right] \\
& +\tau R_{1 i}^{-1}\left[N_{1 i} N_{2 i}\right] \eta_{1}(k) \\
& +r^{-d} \eta_{2}^{T}(k)\left[\begin{array}{l}
M_{1 i}^{T} \\
M_{2 i}^{T}
\end{array}\right] d R_{2 i}^{-1}\left[M_{1 i} \quad M_{2 i}\right] \eta_{2}(k) \\
& +\left(r^{-1}-1\right) V_{i}(k),
\end{aligned}
$$

where 
From (16), (28), and (29), when $k \in\left[k_{m}, k_{m+1}\right)$, there holds

$$
\begin{aligned}
V_{\sigma(k)}(k) & \leq r^{-\left(k-k_{m}\right)} V_{\sigma\left(k_{m}\right)}\left(k_{m}\right) \\
& \leq \mu r^{-\left(k-k_{m}\right)} V_{\sigma\left(k_{m}-1\right)}\left(k_{m}\right) \\
& \leq \mu r^{-\left(k-k_{m}\right)} r^{-\left(k_{m}-k_{m-1}\right)} V_{\sigma\left(k_{m}-1\right)}\left(k_{m-1}\right) \\
& =\mu r^{-\left(k-k_{m-1}\right)} V_{\sigma\left(k_{m}-1\right)}\left(k_{m-1}\right) \\
& \leq \cdots \leq \mu^{N_{\sigma}} r^{-\left(k-k_{0}\right)} V_{\sigma\left(k_{0}\right)}\left(k_{0}\right) .
\end{aligned}
$$

Observe that

$$
N_{\sigma} \leq \frac{k-k_{0}}{T_{a}}, \quad \mu=r^{\ln \mu / \ln r} .
$$

This together with (30) and (33) yields

$$
\mu^{N_{\sigma}} r^{-\left(k-k_{0}\right)} V_{\sigma\left(k_{0}\right)}\left(k_{0}\right) \leq \lambda^{-\left(k-k_{0}\right)} V_{\sigma\left(k_{0}\right)}\left(k_{0}\right) .
$$

This further implies

$$
V_{\sigma(k)}(k) \leq \lambda^{-\left(k-k_{0}\right)} V_{\sigma\left(k_{0}\right)}\left(k_{0}\right) .
$$

Let

$$
\begin{aligned}
& \beta_{1}=\min \left\{\min _{i \in \mathcal{N}}\left\{\lambda_{m}\left(P_{1 i}\right)\right\}, \min _{i \in \mathcal{N}}\left\{\lambda_{m}\left(P_{2 i}\right)\right\}\right\}, \\
& \beta_{2}=\max \left\{\beta_{21}, \beta_{22}\right\},
\end{aligned}
$$

where

$$
\begin{aligned}
\beta_{21}= & \max _{i \in \mathcal{N}}\left\{\lambda_{M}\left(P_{1 i}\right)\right\}+\frac{r\left(1-r^{-\tau}\right)}{r-1} \max _{i \in \mathcal{N}}\left\{\lambda_{M}\left(Q_{1 i}\right)\right\} \\
& +2 \frac{r \tau(r-1)-r\left(1-r^{-\tau}\right)}{(r-1)^{2}} \max _{i \in \mathcal{N}}\left\{\lambda_{M}\left(R_{1 i}\right)\right\}, \\
\beta_{22}= & \max _{i \in \mathcal{N}}\left\{\lambda_{M}\left(P_{2 i}\right)\right\}+\frac{r\left(1-r^{-d}\right)}{r-1} \max _{i \in \mathcal{N}}\left\{\lambda_{M}\left(Q_{2 i}\right)\right\} \\
& +2 \frac{r d(r-1)-r\left(1-r^{-d}\right)}{(r-1)^{2}} \max _{i \in \mathcal{N}}\left\{\lambda_{M}\left(R_{2 i}\right)\right\} .
\end{aligned}
$$

It can be verified from (31) that

$$
\begin{gathered}
V_{\sigma(k)}(k) \geq \beta_{1}\left(\|x(k)\|^{2}+\|y(k)\|^{2}\right), \\
V_{\sigma\left(k_{0}\right)}\left(k_{0}\right) \leq \beta_{21}\|\phi(k)\|_{L}^{2}+\beta_{22}\|\psi(k)\|_{L}^{2},
\end{gathered}
$$

which gives rise to

$$
\begin{aligned}
\beta_{1}\left(\|x(k)\|^{2}+\|y(k)\|^{2}\right) & \leq V_{\sigma(k)}(k) \leq \lambda^{-\left(k-k_{0}\right)} V_{\sigma\left(k_{0}\right)}\left(k_{0}\right) \\
& \leq \lambda^{-\left(k-k_{0}\right)} \beta_{2}\left(\|\phi\|_{L}^{2}+\|\psi\|_{L}^{2}\right) .
\end{aligned}
$$

Therefore, we have

$$
\|x(k)\|^{2}+\|y(k)\|^{2} \leq \frac{\beta_{2}}{\beta_{1}}\left(\|\phi\|_{L}^{2}+\|\psi\|_{L}^{2}\right) \lambda^{-\left(k-k_{0}\right)},
$$

which implies that the discrete-time switched BAM neural networks $(\Sigma)$ are exponentially stable. This completes the proof of Theorem 9.
Remark 10. In (30), the function ceil( $t$ ) is used, which represents rounding real number $t$ to the nearest integer greater than or equal to $t$. The reason that we introduce the function ceil is that the dwell time length of the currently active subsystem is the number of sampling periods between the two consecutive switching times.

Remark 11. In [3-6], the asymptotic or exponential stability problem is considered for continuous-time BAM neural networks with time delay

$$
\begin{gathered}
\dot{x}(t)=-A x(t)+W f(y(t-d)), \\
\dot{y}(t)=-B x(t)+V g(x(t-\tau)) .
\end{gathered}
$$

However, the dynamics of discrete-time neural networks may be quite different from those of continuous-time ones, and the stability criteria established for continuous-time BAM neural networks model are not necessarily applicable to discrete-time systems. Considering the importance in both theory and practice, it is necessary to study the dynamics of the discrete-time BAM neural networks.

Remark 12. There are few references concerning exponential stability analysis for discrete-time switched BAM neural networks. In this paper, some delay-dependent sufficient conditions checking the exponential stability of discrete-time switched BAM neural networks using average dwell time approach are presented. These conditions are proposed in the form of linear matrix inequalities, which can be easily solved by using the recently developed interior algorithms.

\section{An Illustrative Example}

Consider the discrete-time switched BAM neural networks $(\Sigma)$ combining two subsystems with the following parameters:

$$
\begin{gathered}
A_{1}=\left[\begin{array}{cc}
0.5 & 0 \\
0 & 0.5
\end{array}\right], \\
W_{1}=\left[\begin{array}{cc}
-0.0483 & -0.01 \\
-0.03 & -0.04
\end{array}\right], \quad B_{1}=\left[\begin{array}{cc}
0.3 & 0 \\
0 & 0.2
\end{array}\right], \\
V_{1}=\left[\begin{array}{ll}
-0.05 & -0.01 \\
-0.05 & -0.06
\end{array}\right], \\
A_{2}=\left[\begin{array}{cc}
0.7 & 0 \\
0 & 0.4
\end{array}\right], \quad B_{2}=\left[\begin{array}{cc}
0.6 & 0 \\
0 & 0.7
\end{array}\right], \\
W_{2}=\left[\begin{array}{cc}
-0.032 & -0.06 \\
-0.01 & -0.05
\end{array}\right], \quad V_{2}=\left[\begin{array}{cc}
-0.05 & -0.01 \\
-0.05 & -0.06
\end{array}\right] .
\end{gathered}
$$

The activation functions are taken as

$$
\begin{aligned}
& f(y)=\frac{1}{2}(|y+1|-|y-1|), \\
& g(x)=\frac{1}{2}(|x+1|-|x-1|) .
\end{aligned}
$$

Let $d=1$ and $\tau=1$. Solving LMI (17), (28), and (29), it is found that the LMIs are feasible for all $r \leq 1.47$. 
TABle 1: The maximum delay bound $\tau, d$ and decay rate $\lambda$.

\begin{tabular}{|c|c|c|c|c|c|}
\hline$r$ & 1.45 & 1.4 & 1.35 & 1.3 & 1.25 \\
\hline$\lambda$ & 1.2083 & 1.1667 & 1.1250 & 1.0833 & 1.0417 \\
\hline$d=2, \tau_{\max }$ & 10 & 14 & 18 & 22 & 28 \\
\hline$d=3, \tau_{\max }$ & 9 & 13 & 17 & 21 & 27 \\
\hline$d=5, \tau_{\max }$ & 7 & 11 & 15 & 19 & 25 \\
\hline$d=8, \tau_{\max }$ & 4 & 8 & 12 & 16 & 22 \\
\hline$d=11, \tau_{\max }$ & 1 & 5 & 9 & 13 & 19 \\
\hline$d=14, \tau_{\max }$ & - & 2 & 6 & 10 & 16 \\
\hline$d=17, \tau_{\max }$ & - & - & 3 & 7 & 13 \\
\hline$d=20, \tau_{\max }$ & - & - & - & 4 & 10 \\
\hline $\max \{d=\tau\}$ & 6 & 8 & 10 & 12 & 15 \\
\hline
\end{tabular}

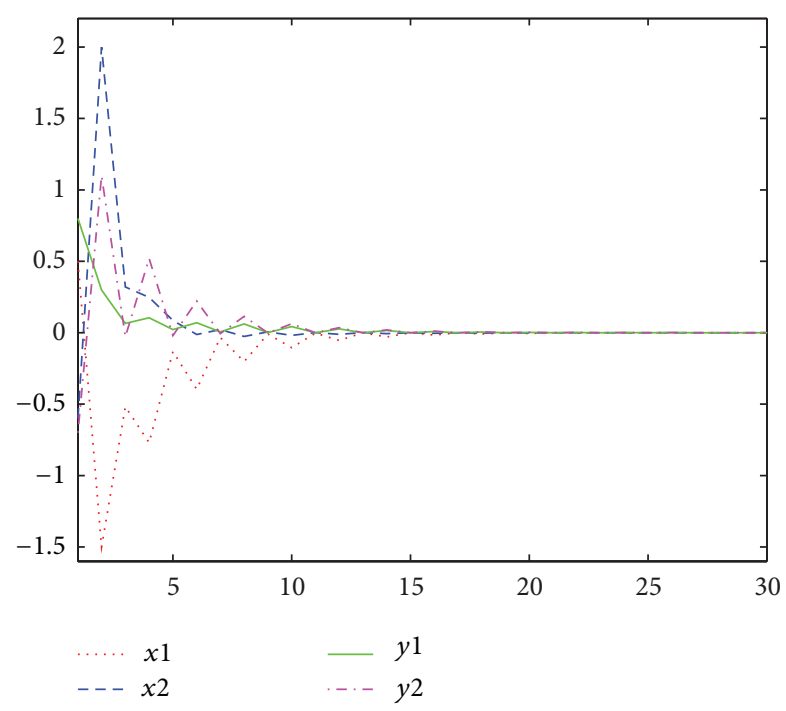

FIGURE 1: State response of the given system.

The calculated values of the delay upper bound $\tau$ and decay rate $\lambda$ for different values of $d$ and $r$ are given in Table 1 when $T_{a}=1$. From Table 1 , we can see that the delay is related to the decay rate. For a given $d$, a smaller decay rate $\lambda$ allows a larger delay $\tau_{\text {max }}$. Moreover, for every $r$, the delay $\tau_{\max }$ decreases when the delay $d$ increases.

Letting $r=1.4, d=3$, and $\mu=1.2$, we obtain that $T_{a}^{*}=$ ceil[0.5419]. Based on (30), $T_{a}=1$ is satisfied. Then we can calculate that the decay rate $\lambda=r^{\left(-\ln \mu / T_{a} \ln r\right)+1}=1.1667$. Therefore, the discrete-time switched BAM neural networks with time delay are exponentially stable with the decay rate $\lambda=1.1667$ if the delay $\tau$ is not larger than 13 based on Table 1.

For $r=1.4, d=3$, and $\tau=2$, based on Definition 3, the discrete-time switched BAM neural networks ensure the following exponential decay estimation:

$$
\begin{aligned}
& \|x(k)\|^{2}+\|y(k)\|^{2} \\
& \quad \leq 2.2866 \times 1.1667^{-\left(k-k_{0}\right)}\left(\|\phi\|_{L}^{2}+\|\psi\|_{L}^{2}\right), \quad \forall k \geq k_{0} .
\end{aligned}
$$

Let $k_{0}=0$. Suppose the switching sequence is: $121212 \ldots$.. It can be seen from switched sequence that $T_{a}=1$. Choose initial value as $\phi(s)=\left[\begin{array}{ll}0.5 & -0.6\end{array}\right]^{T}$ and $\psi(s)=\left[\begin{array}{ll}0.8 & -0.7\end{array}\right]^{T}$; then we obtain Figure 1, which depicts the trajectories of the system state.

\section{Conclusions}

In this paper, the exponential stability problem for the discrete-time switched BAM neural networks with time delay has been proposed. At first, the mathematical model of the discrete-time switched BAM neural networks with time delay has been established. And then by constructing a new switching-dependent Lyapunov-Krasovskii functional, some sufficient criteria have been developed to guarantee the discrete-time switched BAM neural networks to be exponentially stable based on the average dwell time approach and finite sum inequality technology. Finally, a numerical example has been provided to demonstrate the potential and effectiveness of the proposed algorithms.

\section{Acknowledgments}

This work is supported by National Nature Science Foundation of China under Grant 61104132 and Director of the Foundation under Grant SJ201002.

\section{References}

[1] B. Kosko, "Adaptive bidirectional associative memories," Applied Optics, vol. 26, no. 23, pp. 4947-4960, 1987.

[2] B. Kosko, "Bidirectional associative memories," IEEE Transactions on Systems, Man, and Cybernetics, vol. 18, no. 1, pp. 49-60, 1988.

[3] J. H. Park, "A novel criterion for global asymptotic stability of BAM neural networks with time delays," Chaos, Solitons \& Fractals, vol. 29, no. 2, pp. 446-453, 2006.

[4] C. Li, X. Liao, and R. Zhang, "Delay-dependent exponential stability analysis of bi-directional associative memory neural networks with time delay: an LMI approach," Chaos, Solitons \& Fractals, vol. 24, no. 4, pp. 1119-1134, 2005.

[5] G. Zong, L. Hou, and H. Yang, "Further results concerning delay-dependent $H_{\infty}$ control for uncertain discrete-time 
systems with time-varying delay," Mathematical Problems in Engineering, vol. 2009, Article ID 732181, 24 pages, 2009.

[6] G. Zong and J. Liu, "New delay-dependent global robust stability conditions for interval neural networks with time-varying delays," Chaos, Solitons \& Fractals, vol. 42, no. 5, pp. 2954-2964, 2009.

[7] J. Liu and G. Zong, "New delay-dependent asymptotic stability conditions concerning BAM neural networks of neutral type," Neurocomputing, vol. 72, no. 10-12, pp. 2549-2555, 2009.

[8] L. Sheng and H. Yang, "Novel global robust exponential stability criterion for uncertain BAM neural networks with time-varying delays," Chaos, Solitons \& Fractals, vol. 40, no. 5, pp. 2102-2113, 2009.

[9] M. Gao and B. Cui, "Global robust exponential stability of discrete-time interval BAM neural networks with time-varying delays," Applied Mathematical Modelling, vol. 33, no. 3, pp. 12701284, 2009.

[10] J. Liang, J. Cao, and D. W. C. Ho, "Disctete-time bidirectional associative memory neural networks with variable delays," Physicas Letters A, vol. 335, no. 1, pp. 226-234, 2005.

[11] D. Liberzon and A. S. Morse, "Basic problems in stability and design of switched systems," IEEE Control Systems Magazine, vol. 19, no. 5, pp. 59-70, 1999.

[12] G. Zong, L. Hou, and Y. Wu, "Exponential $l_{2}-l_{\infty}$ filtering for discrete-time switched systems under a new framework," International Journal of Adaptive Control and Signal Processing, vol. 26, no. 2, pp. 124-137, 2012.

[13] W.-A. Zhang and L. Yu, "Stability analysis for discrete-time switched time-delay systems," Automatica, vol. 45, no. 10, pp. 22652271, 2009.

[14] M. S. Mahmoud, "Switched discrete-time systems with timevarying delays: a generalized $\mathscr{H}_{2}$ approach," Computers \& Mathematics with Applications, vol. 57, no. 1, pp. 79-95, 2009.

[15] C. Li, G. Feng, and T. Huang, "On hybrid impulsive and switching neural networks," IEEE Transactions on Systems, Man, and Cybernetics, Part B, vol. 38, no. 6, pp. 1549-1560, 2008.

[16] H. Huang, Y. Qu, and H. X. Li, "Robust stability analysis of switched Hopfield neural networks with time-varying delay under uncertainty," Physics Letters A, vol. 345, no. 4-6, pp. 345$354,2005$.

[17] K. Yuan, J. Cao, and H. X. Li, "Robust stability of switched Cohen-Grossberg neural networks with mixed time-varying delays," IEEE Transactions on Systems, Man, and Cybernetics, Part B, vol. 36, no. 6, pp. 1356-1363, 2006.

[18] S. Boyd, L. El Ghaoui, E. Feron, and V. Balakrishnan, Linear Matrix Inequalities in System and Control Theory, vol. 15 of SIAM Studies in Applied Mathematics, SIAM, Philadelphia, Pa, USA, 1994. 


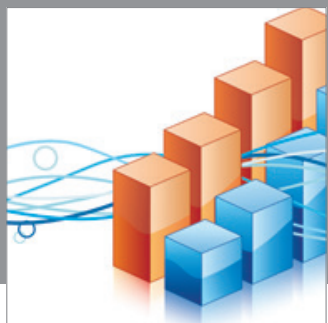

Advances in

Operations Research

mansans

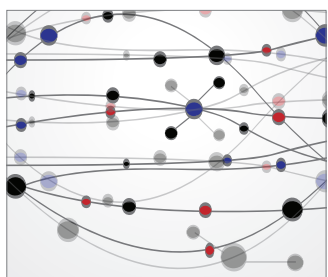

The Scientific World Journal
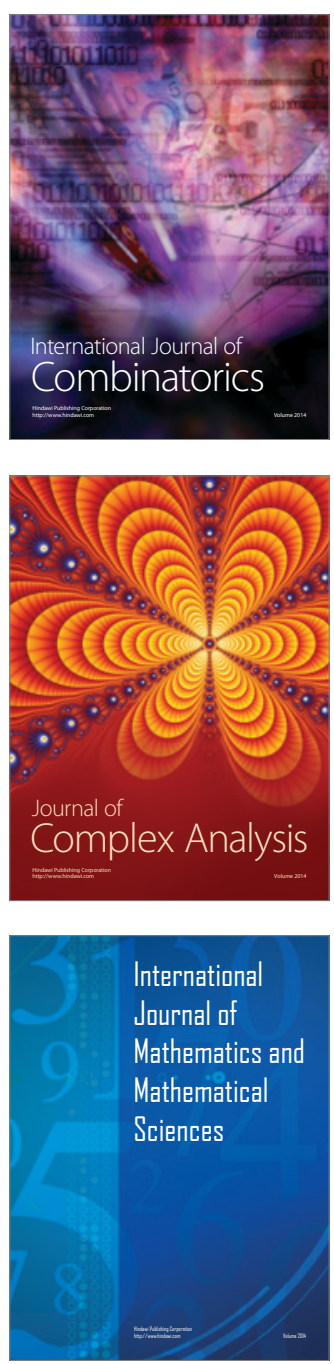
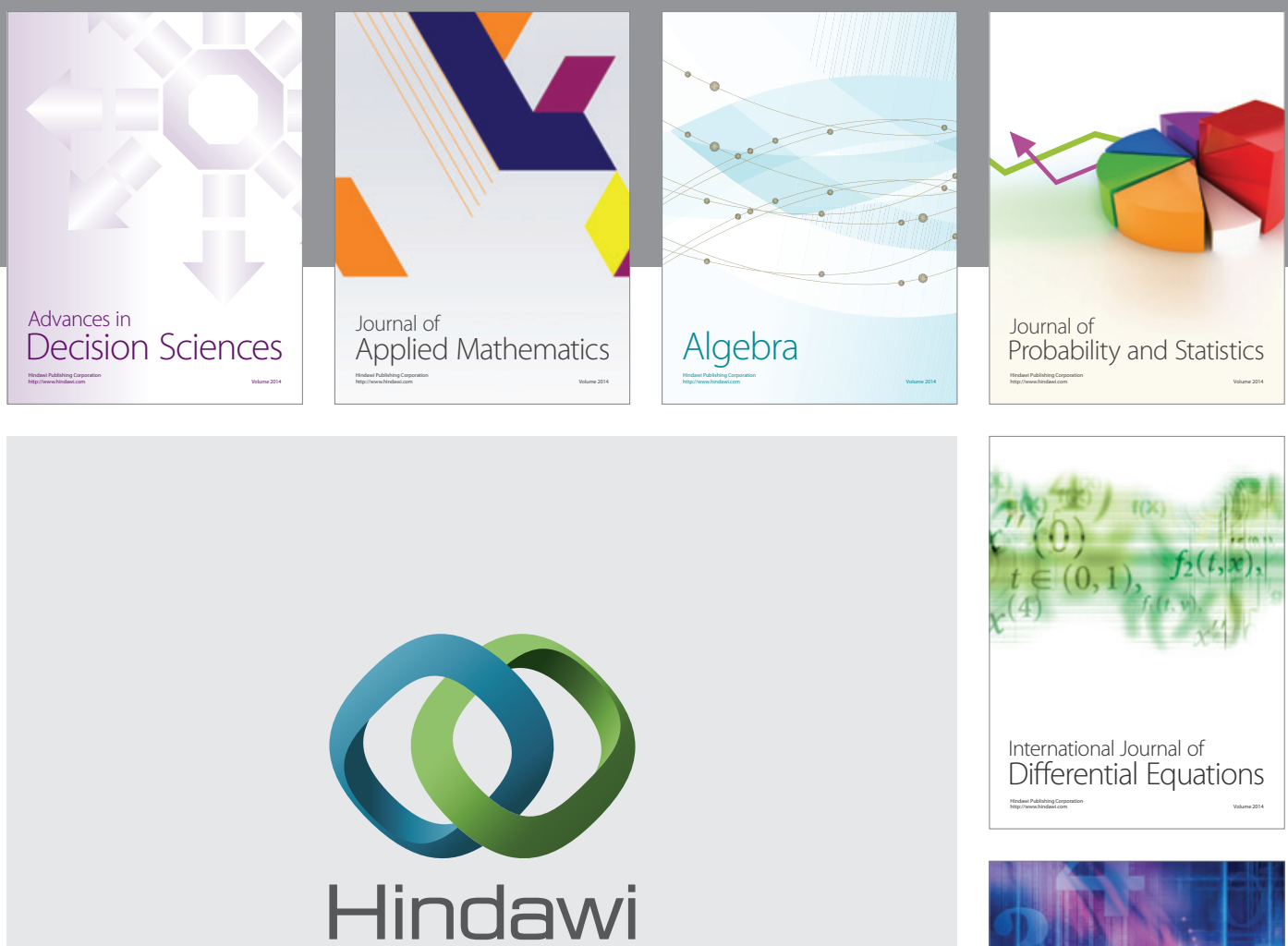

Submit your manuscripts at http://www.hindawi.com
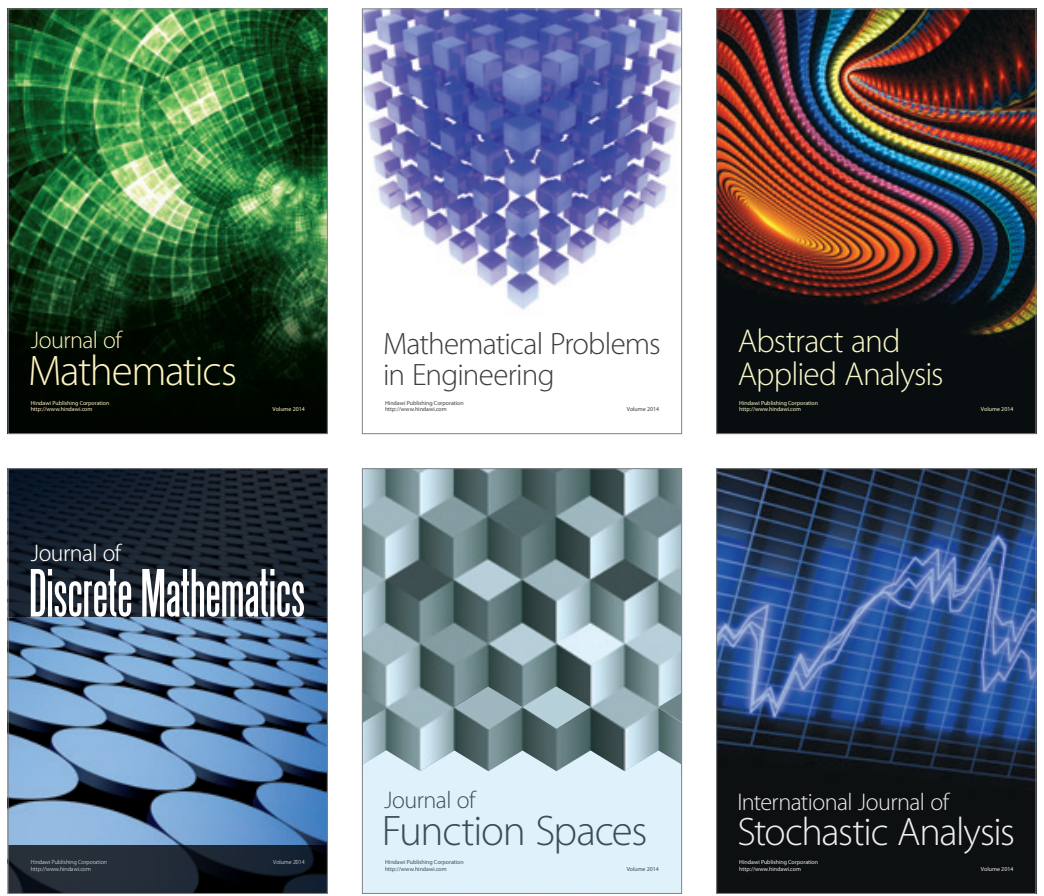

Journal of

Function Spaces

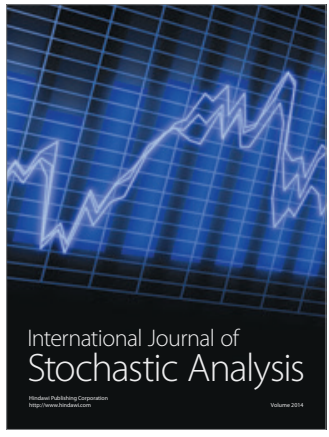

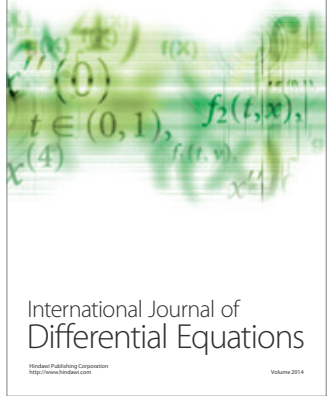
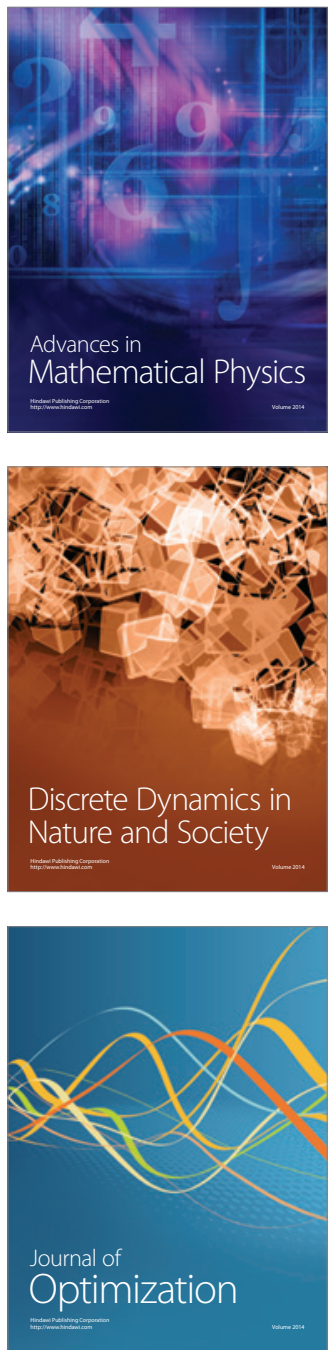